\title{
Urinary chiro- and myo-inositol levels as a biological marker for type 2 diabetes mellitus
}

\author{
Jun Hwa Hong ${ }^{\mathrm{a}, 1}$, Hye Won Jang ${ }^{\mathrm{a}, 1}$, Yea Eun Kang ${ }^{\mathrm{a}}$, Ju Hee Lee ${ }^{\mathrm{a}}$, Koon Soon Kim ${ }^{\mathrm{a}}$, Hyun Jin Kimª, \\ Kyu Ri Park ${ }^{\mathrm{b}}$ and Bon Jeong $\mathrm{Ku}^{\mathrm{a}, *}$ \\ ${ }^{a}$ Department of Internal Medicine, Chungnam National University School of Medicine, Daejeon, Korea \\ ${ }^{\mathrm{b}}$ Daejeon-Chungnam Branch, Korean Association of Health Promotion, Daejeon, Korea
}

\begin{abstract}
Background: The aim of this study was to investigate the role of the urinary chiro- and myo-inositol levels in predicting type 2 diabetes mellitus (T2DM).

Subjects and methods: A total of 212 normal controls and 101 type 2 diabetic patients were enrolled this study. The concentrations of urinary chiro- and myo-inositol were measured by high performance liquid chromatography/mass spectrometry.

Results: The concentration of urinary chiro-inositol was significantly higher in the diabetic subjects $(2.24 \pm 5.18 \mathrm{ng} / \mathrm{L})$ than those in the control group $(0.38 \pm 0.62 \mathrm{ng} / \mathrm{L} ; p<0.001)$. The urinary myo-inositol level of the diabetic subjects $(36.95 \pm 37.77 \mathrm{ng} / \mathrm{L})$ was also significantly higher than that of the controls $(8.17 \pm 13.29 \mathrm{ng} / \mathrm{L} ; p<0.001)$. The urinary chiro-inositol multiplied by myo-inositol level of the diabetic subjects $(148.10 \pm 544.91)$ was significantly higher than in the controls $(5.12 \pm 24.15$; $p<0.001)$. The area under the receiver operating characteristic curve for the urinary chiro-inositol multiplied by myo-inositol level to predict T2DM was 0.840 (confidence interval $0.789-0.891, p<0.001$ ). The cut-off value for the urinary chiro-inositol multiplied by myo-inositol level to predict T2DM was 2.20 (sensitivity $81.3 \%$, specificity $70.3 \%$ ).

Conclusions: The urinary chiro- and myo-inositol concentrations were increased in the type 2 diabetic patients and the urinary chiro- times the myo-inositol was considered to be a sufficient marker in predicting T2DM.
\end{abstract}

Keywords: Chiro-inositol, myo-inositol, diabetes mellitus

\section{Introduction}

The prevalence of diabetes is rapidly increasing. The prevalence of diabetes for all age-groups, worldwide, was estimated to be $2.8 \%$ in 2000 and projected to be $4.4 \%$ by 2030 [1]. In Korea, $9.8 \%$ of all age groups and $22 \%$ of people $>60$ years of age had diabetes, according to the 2009 Korea National Health and Nutrition Examination Survey (KNHANES IV-3). The rate of awareness of people $>30$ years of ages increased to $70 \%$ in 2007 . However, the awareness of age $30 \sim 39$ was only $30 \%$ [2]. Thus, it is important that diabetes mellitus must be detected, while it is in a mild stage and young age.

\footnotetext{
${ }^{1}$ These two authors contributed equally to this article.

* Corresponding author: Bon Jeong Ku, Department of Internal Medicine, Chungnam National University School of Medicine, 282 Munhwa-ro, Jung-gu, Daejeon 301-721, Korea. Tel.: +82 42280 7149; Fax: +82 42280 7995; E-mail: bonjeong@cnu.ac.kr.
}

Many studies tried to estimate the risk for the development of type 2 diabetes. Most researchers have depended on a 2-hour oral glucose tolerance test (OGTT) for identifying impaired glucose tolerance. Previous studies have demonstrated that OGTT-derived measures of insulin resistance and impaired insulin secretion can predict future development of type 2 diabetes $[3,4]$. In contrast, some researchers questioned the reliability of OGTT. Abdul-Ghani et al. [5] reported that the insulin secretion/insulin resistance index was a useful predictor of future development of type 2 diabetes and considered to be more powerful than OGTT. Michael et al. [6] pronounced that persons at high risk for diabetes mellitus were better identified by using a simple prediction model than by relying exclusively on the results of a 2-hour glucose tolerance test. Further, Henry et al. [7] also reported that two-risk scoring systems, which use clinical data and fasting blood tests, were better in identifying the risk for type 2 diabetes. Furthermore, oral glucose test takes a long time, over 
2 hours, high cost, patient's fear or rejection of needle puncture and medical labor. It is required to find out convenient and easy-accessible method for screening.

In the pathogenesis of type 2 diabetes, it has become evident that hyperglycemia is associated with both insulin resistance and beta-cell dysfunction [8]. Some actions of insulin are performed by inositolphosphoglycan (IPG) mediators that are released by cells after stimulation of insulin $[9,10]$. IPG molecules are hydrolyzed from glycosylphosphatidyl-inositols (GPIs) in cell membranes, in response to insulin and are considered putative insulin mediators. IPGs exist as two forms: chiro-inositols and myo-inositols. Majority of IPGs exist as myo-inositols, and myo-inositols are converted to chiro-inositol by insulin.

Previous studies had reported the relationship of IPGs and diabetes. Ostlund et al. [11] found that both type 1 and type 2 diabetic patients had increased urinary D-chiro- and myo-inositol excretion compared with normal subjects. Kawa et al. [12] also found that urinary chiro- and myo-inositol excretion was elevated in diabetic $\mathrm{db} / \mathrm{db}$ mouse and streptozotocin diabetic rat. Other study also reported that in women with polycystic ovarian syndrome, urinary chiro-inositol excretion was elevated and hyperinsulinemia with insulin resistance was detected [13]. Large urinary losses are entirely consistent with deficient chiro-inositol content, which was noted previously in the inositol phosphoglycan fractions purified human diabetic muscle [14]. These studies suggested that a deficiency in a chiroinositol phophoglycan (DCI-IPG) might contribute to insulin resistance in individuals with impared glucose tolerance or type 2 diabetes [14,15]. Thus, a defect in an alternative insulin-signaling pathway, in which IPG acts as a mediator of insulin action, which contributes to the pathophysiology of the insulin resistance of type 2 diabetes.

Measuring urinary excretion of inositols is convenient. Larger urinary excretion of IPGs may be associated with larger defect of serum IPGs, putative insulin mediators, and contribute to development of type 2 diabetes. Thus, the aim of this study is to investigate the role of the urinary chiro- and myo-inositol levels in predicting type 2 diabetes mellitus.

\section{Subjects and methods}

\subsection{Subjects}

Total of 313 subjects, 160 males and 153 females were participated in this study, and they were divid- ed into two groups, type 2 diabetes mellitus and nondiabetic control group. The diabetes group consisted of 101 patients with 53 males and 48 females, with the age ranging from 27 to 83 years old that visited the Chungnam National University Hospital from 2009 to 2010. They were all type 2 diabetes and had taken oral hypoglycemic agents or insulin injections. The control group consisted of 212 subjects, 107 males and $105 \mathrm{fe}$ males with age ranging from 21 to 69 years old. They visited the hospital for medical examination. They had have no history of diabetes mellitus, nor any other accompanying medical diseases and their fasting blood glucose levels were below $100 \mathrm{mg} / \mathrm{dL}$. We excluded subjects with renal dysfunction (serum creatinine $>$ $1.5 \mathrm{mg} / \mathrm{dl}$ or glomerular filtration rate $<60 \mathrm{ml} / \mathrm{min}$ ), clinically significant hepatic disease, abnormal liver enzyme (AST > $100 \mathrm{IU} / \mathrm{L}$ or ALT > $100 \mathrm{IU} / \mathrm{L})$ and history of hospitalization for a major cardiovascular event in previous 3 months. Further, we also excluded subjects with infection and detected malignant patients.

We explained our study to all participants and received written informed consent from all participating subjects. The study protocol was reviewed and approved by the Chungnam National University Institutional Review Board.

\subsection{Laboratory tests}

We prepared blood samples in the morning, after overnight fasting (over 8hrs) and checked fasting plasma glucose, HDL, LDL, Triglyceride, total cholesterol, BUN and creatinine, using a blood chemistry analyzer (Hitachi 747, Tokyo, Japan). Glycosyalted hemoglobin was measured using a high-performance liquid chromatography (Biorad, Hercules, CA, USA). We prepared spot urine soon after blood sampling. The concentrations of urinary chiro- and myo-inositol were measured by high performance liquid chromatography/mass spectrometry (Applied Biosystems $4000 \mathrm{Q}$ TRAP, Foster city, CA, USA).

\subsection{Statistical analysis}

Data was expressed as mean \pm SD. Unpaired Student's $t$-test was used in comparison of baseline characteristics between the two groups. Pearson's correlation was used to assess associations between variables. Statistical significance was considered at the level of $p<$ 0.05 . The comparison of prediction factor for development of type 2 diabetes was tested with the area under the receiver-operating characteristic (ROC) curve. Statistical analysis was performed with the SPSS (version 18.0 for Windows; SPSS Inc., Chicago, IL). 
Table 1

Comparison of anthropometric and metabolic characteristics of the two study groups

\begin{tabular}{|c|c|c|c|}
\hline & Normal $(n=212)$ & Type 2 DM $(n=102)$ & $P$ value \\
\hline Age (yr) & $42.8 \pm 10.1$ & $55.9 \pm 11.3$ & $<0.001$ \\
\hline Sex (Male/Female) & $107 / 105$ & $53 / 48$ & \\
\hline Body Mass Index $\left(\mathrm{kg} / \mathrm{m}^{2}\right)$ & $23.36 \pm 2.74$ & $25.69 \pm 3.95$ & $<0.001$ \\
\hline Fasting Plasma Glucose (mg/dl) & $87.27 \pm 6.75$ & $140.21 \pm 46.71$ & $<0.001$ \\
\hline Triglyceride $(\mathrm{mg} / \mathrm{dl})$ & $106.90 \pm 61.22$ & $159.76 \pm 91.31$ & $<0.001$ \\
\hline HDL-C (mg/dl) & $56.94 \pm 9.09$ & $47.08 \pm 11.91$ & $<0.001$ \\
\hline LDL-C (mg/dl) & $102.66 \pm 26.39$ & $95.13 \pm 30.39$ & 0.034 \\
\hline Total Cholesterol (mg/dl) & $180.96 \pm 30.52$ & $173.70 \pm 40.44$ & 0.079 \\
\hline BUN (mg/dl) & $11.47 \pm 2.97$ & $15.58 \pm 4.69$ & $<0.001$ \\
\hline Creatinine (mg/dl) & $0.86 \pm 0.15$ & $0.97 \pm 0.17$ & $<0.001$ \\
\hline GFR (ml/min) & $85.93 \pm 16.99$ & $70.04 \pm 18.60$ & $<0.001$ \\
\hline Urine chiro-inositol (ng/dl) & $0.38 \pm 0.62$ & $2.24 \pm 5.18$ & $<0.001$ \\
\hline Urine myo-inositol (ng/dl) & $8.17 \pm 13.29$ & $36.95 \pm 37.77$ & $<0.001$ \\
\hline
\end{tabular}

Data are expressed as mean \pm SD. $P$ values were calculated using an unpaired T-test. HDL-C, high density lipoprotein cholesterol; LDL-C, low density lipoprotein cholesterol; BUN, blood urea nitrogen; Cr, creatinine; GFR, glomerular filtration rate.

Table 2

Sensitivity and specificity of urinary chiro- and myo-inositol, chiro-inositol times myo-inositol as a marker for type 2 diabetes mellitus

\begin{tabular}{lccrr}
\hline & Cut-off value (ng/ml) & Sensitivity (\%) & Specificity (\%) & Areas under ROC (95\% CI) \\
\hline Urine chiro-inositol & 0.31 & 70.8 & 69.8 & $0.791(0.740-0.843)$ \\
Urine myo-inositol & 7.62 & 79.2 & 70.3 & $0.828(0.774-0.883)$ \\
Urine chiro-inositol $\times$ Urine myo-inositol & 2.20 & 81.3 & 70.3 & $0.840(0.789-0.891)$ \\
\hline
\end{tabular}

\section{Results}

\subsection{Baseline characteristics of type 2 diabetic group and control group}

Table 1 presented the anthropometric and metabolic characteristics of the two study groups. The diabetic group was older and had higher BMI with elevated triglyceride and LDL-cholesterol, with lower HDL-cholesterol level. Equivalently prepared urine samples from both groups revealed diabetic patients to have approximately 5 times as much urine chiroand myo-inositol concentration. Urine chiro-inositol concentration in non-diabetic and diabetic patients was $0.38 \pm 0.62 \mathrm{ng} / \mathrm{ml}$ and $2.24 \pm 5.18 \mathrm{ng} / \mathrm{ml}$, respectively. Urine myo-inositol concentration in non-diabetic and diabetic patients was $8.17 \pm 13.29 \mathrm{ng} / \mathrm{ml}$ and $36.95 \pm$ $37.77 \mathrm{ng} / \mathrm{ml}$, respectively. Statistical significance was acceptable ( $p<0.001$ for chiro-inositol; $p<0.001$ for myo-inositol).

\subsection{Comparison of markers for type 2 diabetes prediction}

The concentrations of urinary chiro-inositol, myoinositol, and chiro-inositol multiplied by myo-inositol value were considered to be the markers for the prediction of type 2 diabetes. The urinary chiro-inositol multiplied by myo-inositol concentration in the diabet- ic subjects $(148.10 \pm 544.91 \mathrm{ng} / \mathrm{ml})$ was significantly higher than in the controls $(5.12 \pm 24.15 \mathrm{ng} / \mathrm{ml} ; p<$ 0.001 ). The cut-off value to predict type 2 diabetes was $0.31 \mathrm{ng} / \mathrm{dl}$ (sensitivity $70.8 \%$; specificity $69.8 \%$ ) for urinary chiro-inositol and $7.62 \mathrm{ng} / \mathrm{dl}$ (sensitivity $79.2 \%$; specificity $70.3 \%$ ) for urinary myo-inositol. The cut-off value for urine chiro-inositol multiplied by urine myo-inositol concentration was 2.20 (sensitivity $81.3 \%$; specificity $70.3 \%$ ), as presented in Table 2 . The area under the ROC curve was presented in Fig. 1. The area under the ROC curve $(95 \%$ confidence interval) to predict type 2 diabetes was $0.791(0.740-0.843$, $p<0.001)$ for urinary chiro-inositol and $0.828(0.774$ $0.883, p<0.001$ ) for urinary myo-inositol. The value of urinary chiro-inositol multiplied by urinary myoinositol concentration had the greatest area under the ROC curve (0.837, confidence interval 0.787-0.888, $p<0.001$ ). As a cut-off value of 2.20 in urinary chiro-inositol times the urinary myo-inositol concentration, the sensitivity was $81.3 \%$ and the specificity was $70.3 \%$.

\subsection{Comparison of markers that might affect urinary excretion of chiro- and myo-inositol in type 2 diabetes}

Table 3 presented the comparison of markers that might affect urinary chiro- and myo-inositol levels in type 2 diabetes. We checked the relation of urinary ex- 
Table 3

The comparison of markers that might affect urinary chiro- and myo-inositol levels in type 2 diabetes

\begin{tabular}{|c|c|c|c|c|}
\hline & \multicolumn{2}{|c|}{ Urinary chiro-inositol } & \multicolumn{2}{|c|}{ Urinary myo-inositol } \\
\hline & $\mathrm{r}$ & $p$ & $\mathrm{r}$ & $p$ \\
\hline BMI $\left(\mathrm{kg} / \mathrm{m}^{2}\right)$ & 0.011 & 0.873 & -0.056 & 0.421 \\
\hline $\mathrm{FBS}(\mathrm{mg} / \mathrm{dl})$ & 0.396 & 0.081 & 0.302 & $<0.001$ \\
\hline $\operatorname{HbA1C}(\%)$ & 0.286 & 0.125 & 0.324 & $<0.001$ \\
\hline Duration of DM (yr) & -0.070 & 0.508 & 0.130 & 0.208 \\
\hline GFR (ml/min) & -0.156 & 0.131 & -0.144 & 0.159 \\
\hline Myo-/Chiro-inositol & 0.267 & 0.010 & 0.886 & $<0.001$ \\
\hline
\end{tabular}

$P$ values were calculated using a Pearson's correlation. BMI, body mass index; FBS, fasting blood glucose; HbA1C, hemoglobin A1c; GFR, glomerular filtration rate.

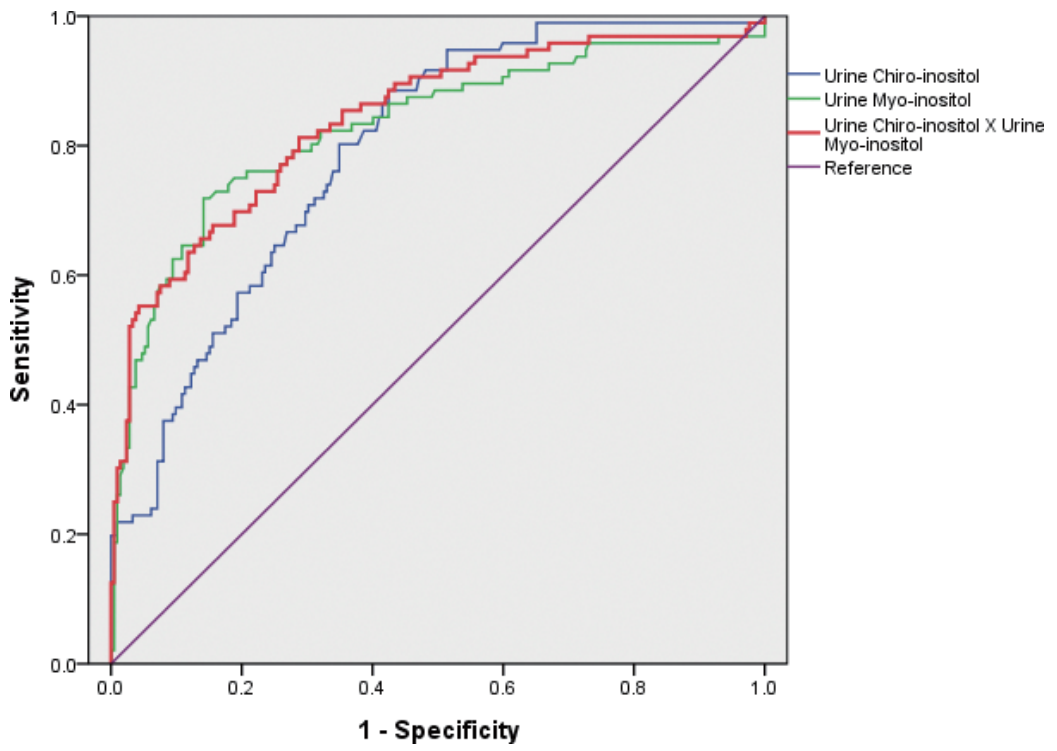

Fig. 1. ROC curves for the diagnostic accuracy of urine chiro-inositol, urine myo-inositol and urine chiro-inositol times urine myo-inositol. (Colours are visible in the online version of the article; http://dx.doi.org/10.3233/DMA-2012-0925)

cretion of chiro- and myo-inositols with obesity. Urinary excretion of chiro-inositol was not correlated with BMI (correlation efficient $=0.011, p=0.873$ ). Urinary excretion of myo-inositol was also not correlated with BMI (correlation efficient $=-0.056, p=0.421$ ). Urinary excretion of myo-inositol showed positive correlation with fasting plasma glucose (correlation coefficient $=0.302, p<0.001$ ) and HbA1c (correlation coefficient $=0.324, p<0.001$ ). However, urinary excretion of chiro-inositol did not show any significant correlation with fasting plasma glucose (correlation coefficient $=0.396, p=0.081)$ and HbA1c (correlation coefficient $=0.286, p=0.125$ ). The duration of type 2 DM showed no correlation with excretion of both chiro- (correlation coefficient $=-0.070, p=0.508$ ) and myo-inositol (correlation coefficient $=0.130, p=$ 0.208).

Renal dysfunction might affect the excretion of inositol. In type 2 diabetes group, there were 28 pa- tients with renal dysfunction (GFR $<60 \mathrm{ml} / \mathrm{min}$ ). Urinary excretion of chiro-inositol was $4.72 \mathrm{ng} / \mathrm{dl}$ in renal dysfunction group and $1.85 \mathrm{ng} / \mathrm{dl}$ in normal renal function group $(p=0.38)$. Urinary excretion of myoinositol was $44.31 \mathrm{ng} / \mathrm{dl}$ in renal dysfunction group and $33.72 \mathrm{ng} / \mathrm{dl}$ in normal renal function group ( $p=$ 0.224). There was no significant difference between urinary excretion of chiro- and myo-inositol and renal dysfunction.

In type 2 diabetes group, there were 28 patients with insulin injection and 73 patients with oral hypoglycemic agent. We divided the group into 2 subgroups by whether they took the insulin injection or not and compared FBS, HbA1c, duration of DM, serum insulin and urine excretions of chiro- and myo-inositol. Patients with insulin injection were more poorly controlled state (more elevated in FBS, HbA1c) and had longer duration of DM. Although the patients with in- 
sulin injection excreted more urine chiro-inositol and urine myo-inositol, serum level of insulin was not significantly different $(p=0.637)$.

Urinary excretion of chiro-inositol showed positive correlation with urinary excretion of myo-inositol (correlation efficient $=0.430, p=0.01$ ). The ratio of urinary excretion of myo-inositol to chiro-inositol presented positive correlation with urinary excretion of myoinositol (correlation coefficient $=0.886, p<0.001$ ) and urinary excretion of chiro-inositol (correlation coefficient $=0.267, p=0.01$ ).

\section{Discussion}

Many researchers presented biologic markers for prediction of type 2 diabetes. Kim et al. [16] suggested HbA1c over $5.95 \%$ for the prediction of type 2 diabetes mellitus with sensitivity $77 \%$ and specificity $89.4 \%$ in Koreans. Marguerite et al. [17] reported that 2-hour glucose by OGTT was useful for predicting diabetes risk, regardless of age. The reference value was 140 $\mathrm{mg} / \mathrm{dl}$ with sensitivity $90 \%$, specificity $68 \%$, and area under ROC curve was 0.829 (0.782-0.876). AbdulGhani et al. [18] also used oral glucose tolerance test, but reported that subjects with 1-hour plasma glucose over $155 \mathrm{mg} / \mathrm{dl}$ present high risk for future diabetes. The 1-hour plasma glucose level had a greatest area under ROC curve $(0.84)$ than that of fasting glucose level (0.75) and 2-hr plasma glucose level (0.79). In our study, the urinary chiro-inositol multiplied by urinary myo-inositol showed an area under ROC curve of $0.837(0.787-0.888)$ and sensitivity $81.3 \%$, specificity $70.3 \%$. Thus, the value of the urinary chiro-inositol multiplied by urinary myo-inositol is suitable for the prediction of type 2 diabetes.

Compared with OGTT, urine analysis is less invasive, has lower cost, requires no medical labor and is convenient. The examination also takes less time, because we examined the concentration of urine chiroand myo-inositol with spot urine, not 24-hrs collected urine. So it is easier to apply to the subjects for the purpose of screening type 2 diabetes.

In concordance with our results, previous studies also reported that urinary excretion of chiro-inositol was elevated in diabetic patients, diabetic $\mathrm{db} / \mathrm{db}$ mouse, streptozotocin diabetic rat and polycystic ovary syndrome patients with increased insulin resistance [1113]. These studies compared groups quantitatively, using $24 \mathrm{hrs}$ urinary excretion of chiro- and myo-inositol. In contrast, we used spot urine to compare the concen- tration of urine chiro- and myo-inositol between the groups. Most of inositols can be obtained from dietary intake of inositol-rich food or synthesized in the body and exist as myo-inositols in the body. Then myoinositols are converted to chiro-inositols by insulin, in the insulin sensitive tissues, such as muscle, liver, and adipose tissues $[19,20]$. Because the serum and urinary chiro-inositol levels change all day long, we obtained urine samples after overnight fasting. In our study, results using spot urine represented the same results, like using 24 hr-collected. Further, GFR of most of all the participants in this study was within the normal range (over $60 \mathrm{ml} / \mathrm{min}$ ). So we suggest that the spot urine can replace the $24 \mathrm{hr}$-collected things.

In contrast to our results, some studies reported decreased urinary excretion of chiro-inositol in conditions that are associated with insulin resistance [14,15,21, 22]. In these studies, urine myo-inositol excretion was increased and urinary chiro-inositol excretion was decreased in the type 2 diabetic subjects. Larner et al. [23] had shown that the increased excretion of urinary myoinositol was due to a competition between glucose and myo-inositol in renal tubular transport. However, the reason of decreased urinary chiro-inositol has not been proven. The difference in the method of measuring the urinary chiro-inositol might be one reason. Some studies used gas chromatography/mass spectrometry inositol assay [21], other studies used quantitative recovery through several purification steps, without internal recovery standard $[14,15]$. In our study, we relied on high performance liquid chromatography/mass spectrometry with the internal standard. We guess that this difference of methodology may attribute to discrepancy of results.

Furthermore, we have performed further analysis to evaluate what factors affected increased urinary excretion of chiro- and myo-inositols in our study. By the Nestler et al., obese women with PCO showed increased clearance on chiro-inositol [24]. We checked the relation of urinary excretion of chiro- and myoinositols with obesity. However, there was no statistically significant correlation between two parameters. In the study of Nestler et al., the BMI of obese group was $36.6 \pm 2.3 \mathrm{~kg} / \mathrm{m}^{2}$ and BMI of non-obese group was $24.2 \pm 2.3 \mathrm{~kg} / \mathrm{m}^{2}$. But in this study, BMI of obese group was only $25.69 \pm 3.96 \mathrm{~kg} / \mathrm{m}^{2}$ and non-obese group $23.36 \pm 2.74 \mathrm{~kg} / \mathrm{m}^{2}$. Renal dysfunction, fasting plasma glucose, HbA1c and duration of type $2 \mathrm{DM}$ might affect the excretion of inositol. In our study, the parameters did not show any correlation with urinary excretion of chiro- and myo-inositol. In type 2 dia- 
betic group, the patients with insulin injection excreted more urinary chiro- and myo-inositol than patients with oral hypoglycemic agent. However, there was no significant difference in serum insulin level. The effect of insulin injection for urinary excretion of chiro- and myo-inositol was needed more studies.

The ratio of urinary excretion of myo-inositol to chiro-inositol presented positive correlation with urinary excretion of myo-inositol and chiro-inositol in this study. We supposed that the overall myo-inositol level might increase in type 2 diabetes patients and the epimerization of myo-inositol to chiro-inositol also increased. And, fasting plasma glucose might significantly affect the urinary excretion of myo-inositol and the epimerization of myo-inositol to chiro-inositol.

Despite of variability in urinary chiro-inositol excretion, most of the studies have shown deficient state of chiro-inositol in human diabetic muscle tissues and of chiro-inositol mediator content and bioactivity [14, 15]. Therefore, we supposed that type 2 diabetes had altered renal metabolism and a large urinary loss of chiro-inositol, which is contributed to increased urinary excretion of chiro-inositol. But the exact mechanism is unknown, so further evaluation and examination are also required to explain such difference.

There was a limitation in our study. We did not control dietary intake. The intake of chiro-inositol mainly depends on diet [19]. Although we prepared the spot urine after overnight fasting, previous diet might affect the urinary excretion.

In conclusion, we presented the value of the urinary chiro-inositol concentration multiplied by urinary myoinositol concentration was a suitable biological marker for the prediction of type 2 diabetes, with the cut-off value of 2.14 (sensitivity $81.3 \%$, specificity $70.3 \%$ ) in this study.

\section{Acknowledgment}

No potential conflicts of interest relevant to this article were reported. This research did not receive any specific grant from any funding agency in the public, commercial or not-for-profit sector.

\section{References}

[1] S. Wild, G. Roglic, A. Green, R. Sicree and H. King, Global prevalence of diabetes: estimates for the year 2000 and projections for 2030, Diabetes care 27 (2004), 1047-1053.
[2] The Fourth Korea National Health and Nutrition Examination Survey (KNHANES IV-3) data, Korea Centers for Disease Control and Prevention, 2010, from http://knhanes.cdc.go.kr/.

[3] R.L. Hanson, R.E. Pratley, C. Bogardus, K.M. Narayan, J.M. Roumain, G. Imperatore, P.H. Benntt, and W.C. Knowler, Evaluation of simple indices of insulin sensitivity and insulin secretion for use in epidemiologic studies, Am J Epidemiol 151 (2000), 190-198.

[4] M.P. Stern, M. Rosenthal, S.M. Haffner, H.P. Hazuda, and L.J. Franco, Sex difference in the effects of sociocultural status on diabetes and cardiovascular risk factors in Mexican Americans. the San Antonio Heart Study, Am J Epidemiol 120 (1984), 834-851.

[5] M.A. Abdul-Ghani, K. William, R.A. DeFronzo, and M. Stern, What is the best predictor of future type 2 diabetes? Diabetes Care 30 (2007), 1544-1548.

[6] M.P. Stern, K. Williams, and S.M. Haffner, Identification of persons at high risk for type 2 diabetes mellitus: Do we need the oral glucose tolerance test? Ann Intern Med 136 (2002), 575-581.

[7] H.S. Kahn, Y.J. Cheng, T.J. Thompson, G. Imperatore, and E.W. Gregg, Two risk-scoring systems for predicting incident diabetes mellitus in U.S. adults age 45 to 64 years, Ann Intern Med 150 (2009), 741-751.

[8] S.E. Kahn, The relative contributions of insulin resistance and beta-cell dysfunction to the pathophysiology of type 2 diabetes, Diabetologia 46 (2003), 3-19.

[9] G. Romero and J. Larner, Insulin mediators and the mechanism of insulin action, Adv Pharmacol 24 (1993), 21-50.

[10] A.R. Saltiel, Second messengers of insulin action, Diabetes Care 13 (1990), 244-256.

[11] R.E. Jr Ostlund, J.B. McGill, I. Herskowitz, D.M. Kipnis, J.V. Santiago, and W. Sherman, D-chiro-inositol metabolism in diabetes mellitus, Proc Natl Acad Sci U S A 90 (1993), 9988-9992.

[12] J.M. Kawa, R. Przybylski, and C.G. Talor, Urinary chiroinositol and myo-inositol excretion is elevated in diabetic $\mathrm{db} / \mathrm{db}$ mouse and streptozotocin diabetic rat, Exp Biol Med 228 (2003), 907-914.

[13] J.P. Baillargeon, E. Diamanti-Kandarakis, R.E. Jr Ostlund, T. Apridonidze, M.J. Iuorno, and J.E. Nestler, Altered D-chiroinositol urinary clearance in women with polycystic ovary syndrome, Diabetes Care 29 (2006), 300-305.

[14] A.S. Kennington, C.R. Hill, J. Craig, C. Bogardus, I. Raz, H.K. Ortmeyer, B.C. Hansen, G. Romero, and J. Larner, Low urinary chiro-inositol excretion in non-insulin-dependent diabetes mellitus, N Engl J Med 323 (1990), 373-378.

[15] I. Asplin, G. Galasko, and J. Larner, chiro-inositol defiency and insulin resistance: A comparison of the chiro-inositol- and myo-inositol-containing insulin mediators isolated from urine, hemodialysate, and muscle of control and type II diabetic subjects, Proc Natl Acad Sci U S A 90 (1993), 5924-5928.

[16] J.H. Kim, G.W. Kim, M.Y. Lee, J.Y. Shin, Y.G. Shin, S.B. Koh, and C.H. Chung, Role of HbA1c in the screening of diabetes mellitus in a Korean rural community, Diabetes Metab J 36 (2012), 37-42.

[17] M.J. McNeely, E.J. Boyko, D.L. Leonetti, S.E. Kahn, and W.Y. Fujimoto, Comparison of a clinical model, the oral glucose tolerance test, and fasting glucose for prediction of type 2 diabetes risk in Japanese Americans, Diabetes Care 26 (2003), 758-763.

[18] M.A. Abdul-Ghani and R.A. Defronzo, Plasma glucose concentration and prediction of future risk of type 2 diabetes, Diabetes Care 32 (2009), 194-198. 
[19] P. Beemster, P. Groenen, and R. Steegers-Theunissen, Involvement of inositol in reproduction, Nutr Rev 60 (2002), 80-87.

[20] Y. Pak, L. Huang, K. Lilley, and J. Larner, In vivo conversion of $\left[{ }^{3} \mathrm{H}\right]$ myo-inositol to $\left[{ }^{3} \mathrm{H}\right]$ chiro-inositol in rat tissues, J Biol Chem 267 (1992), 16904-16910.

[21] J. Larner, D-chiro-inositol - Its functional role in insulin action and its deficit in insulin resistance, Int J Exp Diabetes Res 3 (2002), 47-60.

[22] S. Suzuki, H. Kawasaki, Y. Satoh, M. Ohtomo, M. Matsumoto, M. Hirai, A. Hirai, H. Hirai, S. Hirai, M. Onoda, M. Matsumoto, Y. Hinikio, H. Akai, J. Craig, J. Larner, and T. Toy- ota, Urinary chiro-inositol excretion is an index marker of insulin sensitivity in Japanese type II diabetes, Diabetes Care 17 (1994), 1465-1468.

[23] W.H. Daughaday and J. Larner, The renal excretion of inositol in normal and diabetic human beings, J Clin Invest 33 (1954), 326-332.

[24] Baillargeon JP, Luorno MJ, Apridonidze T, Nestler JE, Uncoupling between insulin and release of a D-chiro-inositolcontaining inositolphosphoglycan mediator of insulin action in obese women with polycystic ovary syndrome, Metab Syndr Relat Disord 8 (2010), 127-136. 


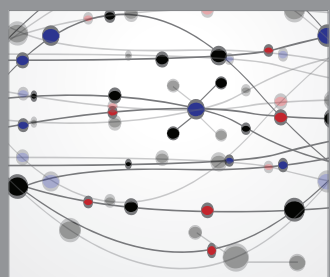

The Scientific World Journal
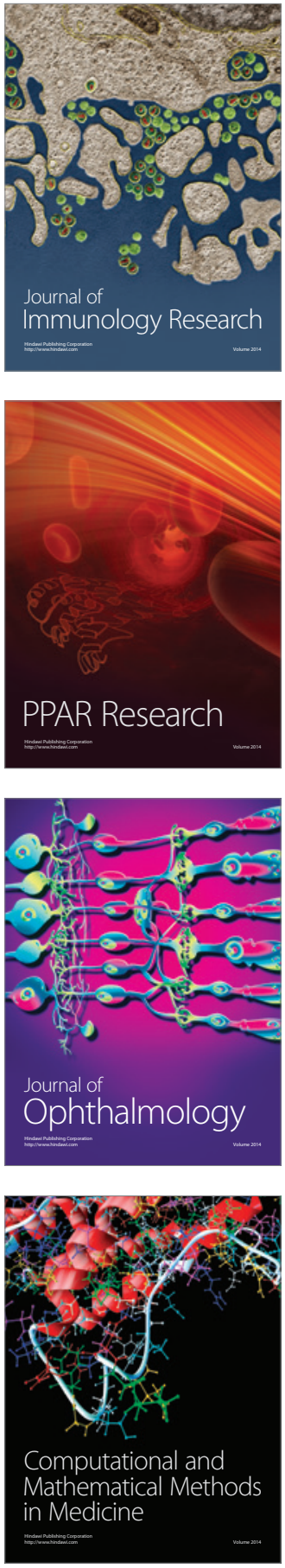

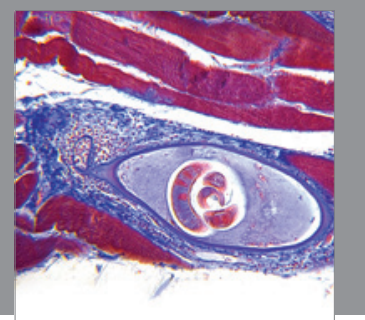

Gastroenterology

Research and Practice
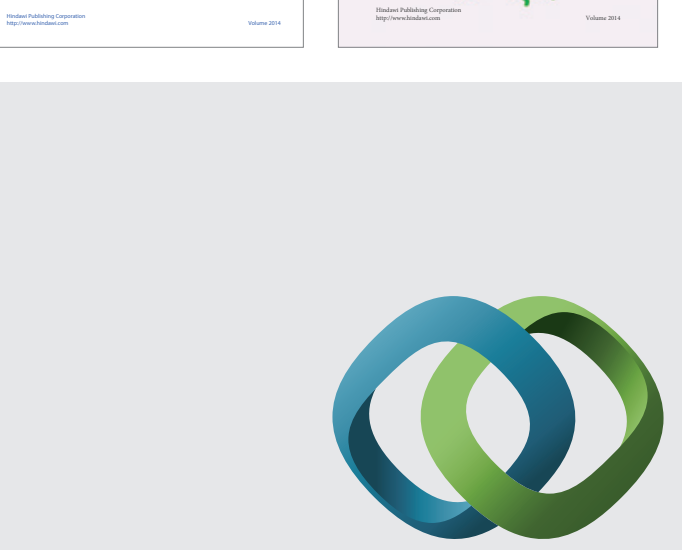

\section{Hindawi}

Submit your manuscripts at

http://www.hindawi.com
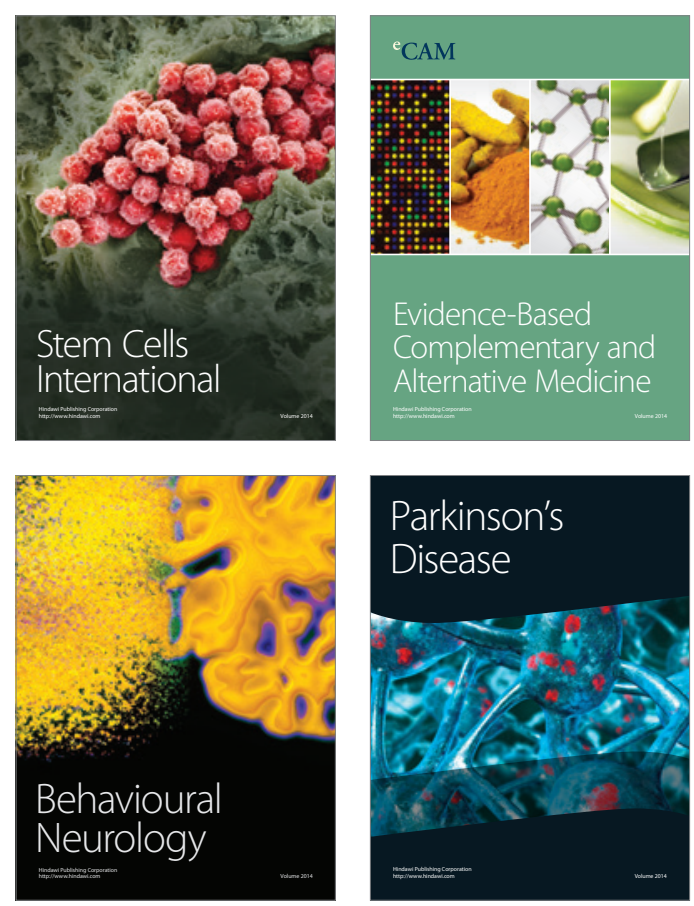

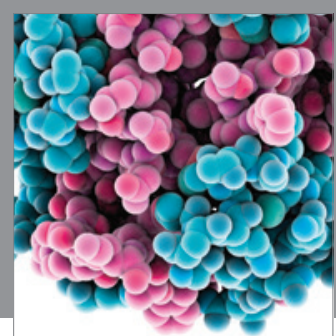

Journal of
Diabetes Research

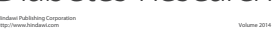

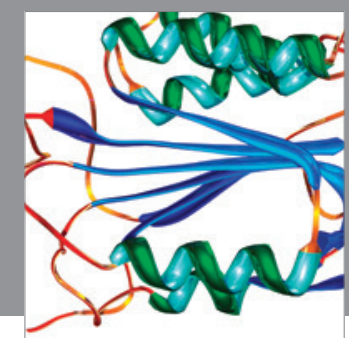

Disease Markers
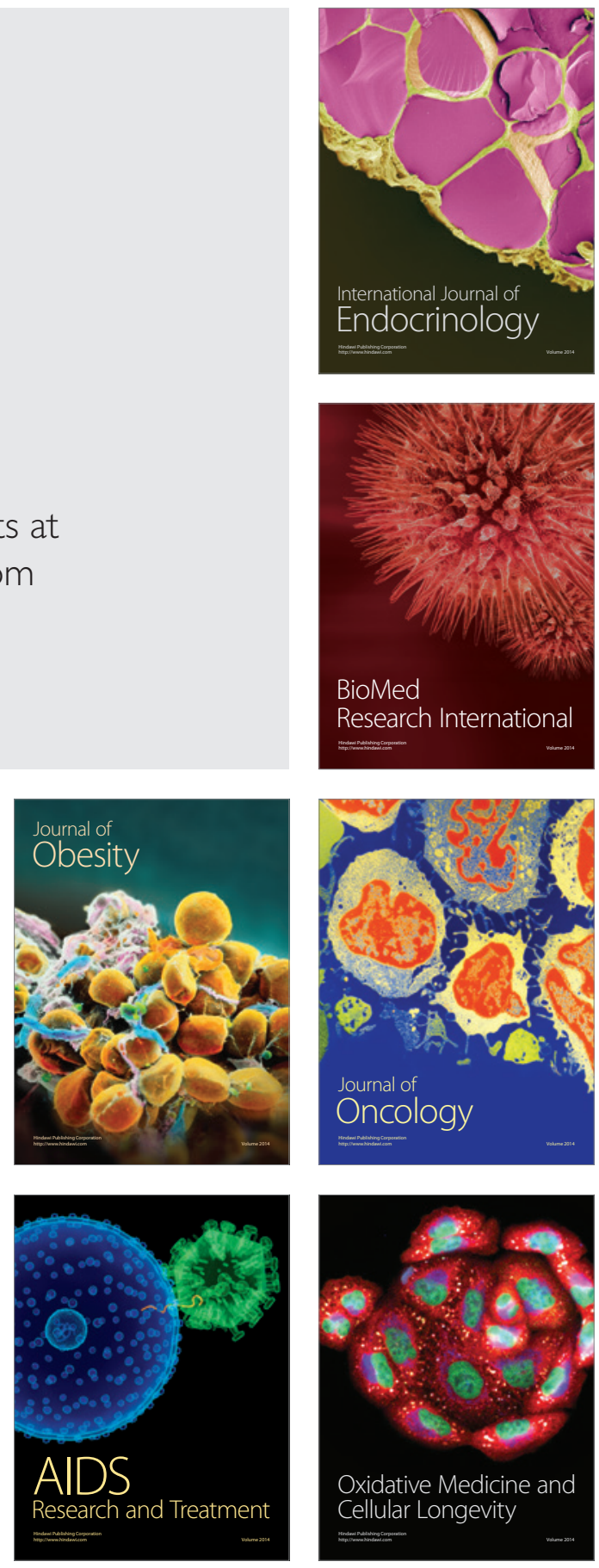